\title{
ON KAEHLERIAN TORSE-FORMING VECTOR FIELDS
}

\author{
By SEIICHI YAMAGUCHI
}

§1. Introduction. K. Yano has studied in [7] the concurrency of a direction defined along a curve $x^{h}(s)$ in $M$, when it satisfies the differential equations

$$
\frac{d x^{h}}{d s}+\frac{\delta \alpha v^{h}}{d s}=0 \text {, }
$$

where $\alpha$ is a suitable function of s. Moreover, generalizing these concepts of parallelism and concurrency, K. Yano [8] has introduced the notion of torseforming directions in $M$ as follows: Consider a vector field $v(s)$ defined along a curve $x^{h}(s)$. If, after the development, the directions defined by $v(s)$ form a developable surface or torse, the directions defined by $v(s)$ are called torse-forming along the curve in $M$.

In order that the directions $v(s)$ defined along a curve $x^{h}(s)$ be torse-forming, it is necessary and sufficient that

$$
\frac{d x^{h}}{d s}+\frac{\delta \alpha v^{h}}{d s}=\beta v^{h},
$$

$\beta$ being another suitable function of the parameter s. A vector field which is always torse-forming along any curve traced in $M$ is called a torse-forming vector field. As for such a vector field, we have known the following theorems [8];

THEOREM A. In order that a Riemannian manifold $M$ admits a torse-forming vector field, it is necessary and sufficient that $M$ contains a family of $\infty^{1}$ totally umbilical hypersurfaces whose orthogonal trajectories are geodesics.

THEOREM B. In order that a Riemannian manifold $M$ admits a torse-forming vector field, it is necessary and sufficient that there exists a coordinate system with respect to which the fundamental quadratıc differential form may be written in the form

$$
\begin{gathered}
d s^{2}=f\left(x^{h}\right) g_{a b}\left(x^{c}\right) d x^{a} d x^{b}+d x^{n} d x^{n} \\
(a, b, c=1,2, \cdots, n-1) .
\end{gathered}
$$

The complex analogue of a torse-forming vector field is, as far as we know, not yet studied. So it might be interesting to develope complex versions of the theory of torse-forming vector fields. In $\S 2$, let us recall first of all definitions and formulas concerning Kaehlerian manifolds and hypersurfaces in a Kaehlerian

Received September 24, 1977. 
manifold for later use. We shall introduce in $\$ 3$ the notion of a Kaehlerian torse-forming vector field along a curve, and investigate in $\$ 4$ a Kaehlerian torse-forming vector field along any curve, which will be called for simplicity a $K$-torse-forming vector field. $\S 5$ is devoted to establish some formulas for later use. In $\S 6$, a kind of hypersufaces called $f$-hypersurfaces will be defined and prove Theorems 4 and 5 . Some examples of Kaehlerian manifolds admitting a $K$-torse-forming vector field will be given in $\S 7$.

$\S 2$. Preliminaries. Let $M$ be a real $2 n$-dimensional Kaehlerian manifold from now on. Denote by $g_{j i}$ and $J_{\jmath}{ }^{h}(h, i, \jmath, \cdots=1,2, \cdots, 2 n)$ the componentes of the Hermitian metric tensor $g$ and those of the complex structure tensor $J$ of $M$ respectively. Then we have by definition

$$
J_{j}^{r} J_{r}{ }^{2}=-\delta_{j}{ }^{2}, \quad g_{j i}=J_{\jmath}^{r} J_{i}{ }^{s} g_{r s}{ }^{1)}, \quad \nabla_{h} J_{\jmath}{ }^{2}=0,
$$

$\nabla$ being the operator of covariant derivation with respect to the Riemannian connection defined by $g$.

The Kaehlerian manifold $M$ is called a space of constant holomorphic sectional curvature if the curvature tensor of $M$ has components of the form

$$
R_{k j i}{ }^{h}=\frac{K}{4}\left(\delta_{k}{ }^{h} g_{j i}-\delta_{\jmath}{ }^{h} g_{k \imath}+J_{k}{ }^{h} J_{j i}-J_{\jmath}{ }^{h} J_{k i}-2 J_{k \jmath} J_{\imath}{ }^{h}\right) \text {. }
$$

Next we shall recall definitions and terminologies in the theory of hypersurfaces in a Kaehlerian manifold. Let us consider a $(2 n-1)$-dimensional orientable submanifold $M^{\prime}$ differentiably immersed in $M$. We fix orientation of $M$ and $M^{\prime}$ and take an open covering $\left\{U_{\beta}\right\}(\beta \in \Lambda)$ of $M$ by coordinate neighborhoods and an open covering $\left\{V_{\alpha}\right\}(\alpha \in \Lambda)$ of $M^{\prime}$ by coordinate neighborhoods so that they are coherent with the orientations, namely, in each coordinate neighborhoods $U_{3}$ of $M$ and $V_{\alpha}$ of $M^{\prime}$ natural frames determine positive positive orientations of those manifolds. Now, each non-empty set $U_{\beta} \cap V_{\alpha}$ can be expressed parametrically as $x^{h}=x^{h}\left(u^{a}\right)(a, b, c, \cdots=1,2, \cdots, 2 n-1)$, where $\left\{x^{h}\right\}$ are local coordinates in $U_{\beta}$ and $\left\{u^{a}\right\}$ are those in $V_{\alpha}$. We now put

$$
B_{a}^{h}=\frac{\partial x^{h}}{\partial u^{a}} \text {. }
$$

Then $B$ are linearly independent local vector fields tangent to $M^{\prime}$. The induced Riemannian metric $g^{\prime}$ of $M^{\prime}$ is given by

$$
g^{\prime}{ }_{a b}=B_{a}{ }^{h} B_{b}{ }^{2} g_{h \imath} .
$$

The manifolds $M$ and $M^{\prime}$ being both orientable, we can choose a unit normal vector field $C^{h}$ along $M^{\prime}$ in such a way that $(C, B)$ determine a frame having the positive sense of $M$ on each non-empty $U_{\beta} \cap V_{\alpha}$. Then we get

1) We adapt the identification between vector fields and 1-forms by virtue of Riemannian metric. 


$$
g_{j i} B_{a}{ }^{\jmath} C^{\imath}=0, \quad g_{j i} C^{\jmath} C^{\imath}=1 .
$$

The transform $J B$ of $B$ by $J$ and $J C$ of $C$ by $J$ are expressed as linear combinations of $B$ and $C$ as follows:

$$
J_{\imath}{ }^{h} B_{a}{ }^{\imath}=\varphi_{c}{ }^{b} B_{b}{ }^{h}+\eta_{b} C^{h}, \quad J_{\imath}{ }^{h} C^{\imath}=-\eta^{a} B_{a}{ }^{h},
$$

because $J C$ is tangent to $M^{\prime}$. It follows from (2.1) and (2.5) that

$$
\begin{gathered}
\varphi_{c}^{b} \varphi_{b}^{a}=-\delta_{c}{ }^{a}+\eta_{c} \eta^{a}, \quad \varphi_{a}^{b} \eta^{a}=0, \\
\eta_{a} \eta^{a}=1 .
\end{gathered}
$$

This means that $M^{\prime}$ admits an almost contact metric structure $\left(\varphi, \eta, g^{\prime}\right)$.

Denoting by $\nabla^{\prime}$ the symbol of the covariant derivation along $M^{\prime}$, we have the equations of Gauss and Weingarten:

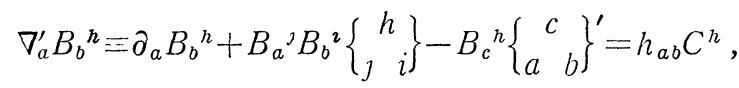

$$
\begin{aligned}
& \nabla_{a}^{\prime} C^{h} \equiv \partial_{a} C^{h}+B_{a}{ }^{\imath} C^{\jmath}\left\{\begin{array}{c}
h \\
j
\end{array}\right\}=-h_{a}{ }^{b} B_{b}{ }^{h},
\end{aligned}
$$

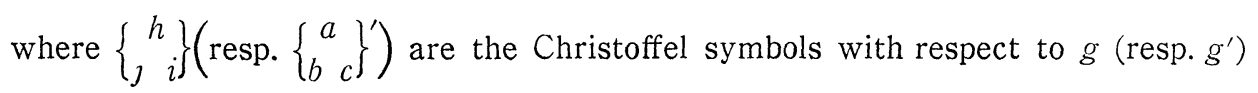
and $h_{a b}$ are components of the second fundamental form of $M^{\prime}$.

When the second fundamental form $h$ of $M^{\prime}$ has the form

$$
h_{a b}=\alpha g_{a b}+\beta \eta_{a} \eta_{b},
$$

$\alpha$ and $\beta$ being certain functions along $M^{\prime}$, then we say that the almost contact contact metric hypersurface $M^{\prime}$ is contact umbilic. As for such $M^{\prime}$, it is well known that a necessary and sufficient condition for an almost contact hypersurface $M^{\prime}$ to be normal and contact metric is that it is contact umbilic $[4,10]$.

§3. Kaehlerian torse-forming vector field along a curve. In what follows $M$ is assumed to be a $2 n$-dimensional Kaehlerian manifold. Let $\xi(s)$ be a vector field along a curve $x^{h}(s)$ in $M$. Such a vector field $\xi(s)$ will be said to be Kaehlerian torse-forming, if the differential equation

$$
\frac{d x^{h}}{d s}+\frac{\delta\left(\alpha \xi^{n}+\beta \tilde{\xi}^{h}\right)}{d s}=\alpha^{\prime} \xi^{n}+\beta^{\prime} \tilde{\xi}^{h}
$$

holds along the curve for any functions $\alpha$ and $\beta$ of the parameter $s, \alpha^{\prime}$ and $\beta^{\prime}$ being certain functions of $s$, where we have put $\tilde{\xi}^{h}=J_{r}^{h} \xi^{r}$. If $\alpha=\beta=0$, then $\xi$ is contained in the section spaned by $d x^{h} / d s$ and $J_{r}{ }^{n} d x^{r} / d s$. If we have $\alpha^{2}+\beta^{2}$ $\neq 0$, then we have from $(3.1)$ 


$$
\frac{\delta \xi^{h}}{d s}=a \frac{d x^{h}}{d s}+b J_{r}{ }^{h} \frac{d x^{r}}{d s}+\lambda \xi^{h}+\mu \tilde{\xi}^{h}
$$

for certain functions $a, b, \lambda$ and $\mu$ along the cueve. We now note that $\alpha^{2}+\beta^{2} \neq 0$ if and only if $a^{2}+b^{2} \neq 0$.

Coversely, if a vector field $\xi(s)$ defined along a curve $x^{h}(s)$ satisfies the differential equations of the form (3.2) with $a$ and $b$ satisfied $a^{2}+b^{2} \neq 0$, then it is easily verified that $\xi(s)$ satisfies a differential equation of the form (3.1). Thus we have

THEOREM 1. Let $\xi(s)$ be a vector field defined along a curve $x^{h}(s)$ and not contained in the section spaned by $d x^{h} / d s$ and $J_{r}^{h} d x^{r} / d s$. Then in order that $\xi(s)$ be a Kaehlerian torse-forming vector field along the curve $x^{h}(s)$, it is necessary and sufficient that the covariant derivative of $\xi(s)$ along the curve be a linear combination of $\xi, \tilde{\xi}, d x^{h} / d s$ and $J_{r}^{h} d x^{r} / d s$.

When $\xi(s)$ satisfies a differential equation (3.2) with $a=b=0$, the two-dimensional distribution spaned by $\xi$ and $\tilde{\xi}$ is parallel.

$\S 4$. K-torse-forming vector field. In this paragraph, let us introduce first of all the notion of a Kaehlerian torse-forming vector field in $M$.

If a vector field $\xi$ satisfies a differential equation of the form (3.2) along any curve traced in $M$, then we call such a vector field $\xi$ a Kaehlerian torse-forming vector field, simply a $K$-torse-forming vector field. Since the equation (3.2) can be rewritten as follows:

$$
\frac{d x^{r}}{d s} \nabla_{r} \xi^{h}=a \frac{d x^{h}}{d s}+b J_{r}{ }^{h} \frac{d x^{r}}{d s}+\lambda \xi^{h}+\mu \tilde{\xi}^{h},
$$

it is easy seen that for a $K$-torse-forming vector field

$$
\nabla_{j} \xi^{h}=a \delta_{j}{ }^{h}+b J_{j}{ }^{h}+\alpha_{j} \xi^{h}+\beta_{j} \tilde{\xi}^{h},
$$

or equivalently

$$
\nabla_{j} \tilde{\xi}^{h}=a J_{j}{ }^{h}-b \delta_{j}{ }^{h}+\alpha_{j} \tilde{\xi}^{h}-\beta_{j} \xi^{h}
$$

for suitable functions $a$ and $b$ and 1-forms $\alpha$ and $\beta$. The functions $a$ and $b$ (resp. 1-forms $\alpha$ and $\beta$ ) appearing in (4.2) will be called the associated functions (resp. forms) of $\xi$. Moreover if the associated functions $a$ and $b$ satisfy $a^{2}+b^{2} \neq 0$ in $M$, then we call such a vector field a proper $K$-torse-forming vector field.

We are now going to obtain some identities containing a $K$-torse-forming vector field for later use. Operating $\nabla_{k}$ to (4.2) and making use of (4.2) and (4.2)', we can easily obtain

$$
\begin{aligned}
\nabla_{k} \nabla_{j} \xi^{h}= & a_{k} \delta_{j}{ }^{h}+b_{k} J_{j}{ }^{h}+\nabla_{k} \alpha \xi^{h}+\nabla_{k} \beta_{j} \tilde{\xi}^{h} \\
& +\alpha_{j}\left(a \delta_{k}{ }^{h}+b J_{k}{ }^{h}+\alpha_{k} \xi^{h}+\beta_{k} \tilde{\xi}^{h}\right)+\beta_{j}\left(a J_{k}{ }^{h}-b \delta_{k}{ }^{h}+\alpha_{k} \tilde{\xi}^{h}-\beta_{k} \xi^{h}\right),
\end{aligned}
$$


from which

$$
\begin{aligned}
R_{k \jmath r}{ }^{h} \xi^{r}= & X_{k} \delta_{\jmath}{ }^{h}-X_{j} \delta_{k}{ }^{h}+Y_{k} J_{\jmath}{ }^{h}-Y_{\jmath} J_{k}{ }^{h} \\
& +\left(\nabla_{k} \alpha_{j}-\nabla_{j} \alpha_{k}\right) \xi^{h}+\left(\nabla_{k} \beta_{j}-\nabla_{j} \beta_{k}\right) \tilde{\xi}^{h},
\end{aligned}
$$

where we have put

$$
X_{k}=a_{k}-a \alpha_{k}+b \beta_{k}, \quad Y_{k}=b_{k}-b \alpha_{k}-a \beta_{k}, \quad a_{k}=\nabla_{k} a, \quad b_{k}=\nabla_{k} b .
$$

Concerning $K$-torse-forming vector fields in a space of constant holomorphic sectional curvature, we have

Proposition 2. In a space $M$ of dimensions $2 n$ ( $>4)$ with constant holomorphic sectional curvature $K$, for any non-vanishing $K$-torse-formm vector field $\xi$ its associated form $\alpha$ is locally a gradient of function and $d \beta=(K / 4) \Phi$, where $\Phi$ is the fundamental two form of Kaehlerian structure of $M$.

Proof. Substituting (2.2) into (4.4), we have

$$
X^{\prime}{ }_{k} \delta_{j}{ }^{h}-X^{\prime}{ }_{j} \delta_{k}{ }^{h}+Y^{\prime}{ }_{k} J_{j}{ }^{h}-Y^{\prime}{ }_{j} J_{k}{ }^{h}+\alpha_{k j} \xi^{h}+\beta_{k j} \tilde{\xi}^{h}=0,
$$

where we have put

$$
\left\{\begin{array}{l}
X_{k}^{\prime}=X_{k}+(K / 4) \xi_{k}, \quad Y_{k}^{\prime}=Y_{k}-(K / 4) \tilde{\xi}_{k}, \\
\alpha_{k \jmath}=\nabla_{k} \alpha_{j}-\nabla_{j} \alpha_{k}, \quad \beta_{k \jmath}=\nabla_{k} \beta_{j}-\nabla_{j} \beta_{k}+(K / 2) J_{k \jmath} .
\end{array}\right.
$$

Hence, since $\operatorname{dim} M>4$, we can take unit vectors $y$ and $\tilde{y}$ in such a way that $y, \tilde{y}, \xi$ and $\tilde{\xi}$ are mutually perpendicular. So, contracting (4.6) with $y_{h}, \tilde{y}_{h}, \xi_{h}$ and $\tilde{\xi}_{h}$, we get by a straightforward computation respectively

$$
\begin{gathered}
X^{\prime}{ }_{k} y_{j}-X^{\prime}{ }_{j} y_{k}-Y^{\prime}{ }_{k} \tilde{y}_{j}+Y^{\prime}{ }_{j} \tilde{y}_{k}=0, \\
X^{\prime}{ }_{k} \tilde{y}_{j}-X^{\prime}{ }_{j} \tilde{y}_{k}+Y^{\prime}{ }_{k} y_{j}-Y^{\prime}{ }_{j} y_{k}=0, \\
X^{\prime}{ }_{k} \xi_{j}-X^{\prime}{ }_{j} \xi_{k}-Y^{\prime}{ }_{k} \tilde{\xi}_{j}+Y^{\prime}{ }_{j} \tilde{\xi}_{k}+\alpha_{k j}|\xi|^{2}=0, \\
X^{\prime}{ }_{k} \tilde{\xi}_{j}-X^{\prime}{ }_{j} \tilde{\xi}_{k}+Y^{\prime}{ }_{k} \xi_{j}-Y^{\prime}{ }_{j} \xi_{k}+\beta_{k j}|\xi|^{2}=0 .
\end{gathered}
$$

From (4.8) and (4.9) it is evident that

$$
\begin{cases}X^{\prime}{ }_{k}-X^{\prime}(y) y_{k}+Y^{\prime}(y) \tilde{y}_{k}=0, & Y^{\prime}{ }_{k}-X^{\prime}(y) \tilde{y}_{k}-Y^{\prime}(y) y_{k}=0, \\ X^{\prime}{ }_{k}-X^{\prime}(\tilde{y}) \tilde{y}_{k}-Y^{\prime}(\tilde{y}) y_{k}=0, & Y^{\prime}{ }_{k}-Y^{\prime}(\tilde{y}) \tilde{y}_{k}+X^{\prime}(\tilde{y}) y_{k}=0,\end{cases}
$$

where we have put $X^{\prime}(y)=X^{\prime}{ }_{k} y^{k}$ etc.. Transvecting (4.12) with $\tilde{y}^{k}, \xi^{k}$ and $\tilde{\xi}^{k}$, we find respectively

$$
\begin{gathered}
Y^{\prime}(\xi)=Y^{\prime}(\tilde{\xi})=X^{\prime}(\xi)=X^{\prime}(\tilde{\xi})=0, \\
X^{\prime}(\tilde{y})+Y^{\prime}(y)=0, \quad X^{\prime}(y)-Y^{\prime}(\tilde{y})=0 .
\end{gathered}
$$


On the other hand, by contraction over $h$ and $\imath$ in (4.6), we can easily verify

$$
(2 n-1) X^{\prime}{ }_{k}+Y^{\prime}{ }_{k}+\alpha_{k r} \xi^{r}+\beta_{k r} \tilde{\xi}^{r}=0 .
$$

Further we transvect (4.10) (resp. (4.11)) with $\xi^{\jmath}$ (resp. $\tilde{\xi}^{\jmath}$ ) and take account of (4.13) so that we obtain

$$
X^{\prime}{ }_{k}+\alpha_{k r} \xi^{r}=0, \quad X^{\prime}{ }_{k}+\beta_{k r} \tilde{\xi}^{r}=0,
$$

which and (4.15) imply

$$
(2 n-3) X^{\prime}{ }_{k}+Y^{\prime}{ }_{k}=0 .
$$

Since $n>2$, this together with (4.14) gives

$$
X^{\prime}(y)=X^{\prime}(\tilde{y})=Y^{\prime}(y)=Y^{\prime}(\tilde{y})=0,
$$

which and (4.12) imply $X^{\prime}{ }_{k}=Y^{\prime}{ }_{k}=0$. Thus (4.6) implies $\alpha_{k j}=\beta_{k j}=0$. Accordingly, Proposition 2 is proved.

For the compact case, we have

THEOREM 3. Let $M$ be a $2 n(>4)$ dimensional compact space of constant holomorphac sectional curvature $K \neq 0$. Then a K-torse-forming vector field in $M$ vanushes identrcally.

Proof. We assume that $\xi$ is a non-vanishing $K$-torse-forming vector field in M. Then by Proposition 2 we obtain

$$
\nabla_{k} \beta_{j}-\nabla_{j} \beta_{k}+(K / 2) J_{k j}=0 .
$$

Contracting this with $J^{k}$, we get $\nabla_{r} \tilde{\beta}^{r}=n K / 2$, from which we have by Green's Theorem

$$
\int_{M} K d V=0,
$$

where $d V$ denotes the volume element of $M$. Thus we have $K=0$. This completes the proof.

§5. Analytic K-torse-forming vector field. From now on suppose that a $K$-torse-forming vector field $\xi$ in $M$ is contravariant analytic. Then the vector field $\xi$ must satisfy (4.2) and

$$
\nabla, \xi_{k}=J_{\jmath}^{r} J_{k}^{s} \nabla_{r} \xi_{s} .
$$

We can easily see that in order that for a $K$-torse-forming vector field $\xi$ to be analytic it is necessary and sufficient that $\beta_{j}=\tilde{\alpha}_{j}\left(=-J_{j}^{r} \alpha_{r}\right)$ holds. Since $\xi$ is analytic, (4.2), (4.3), (4.4) and (4.5) reduce respectively to

$$
\nabla_{j} \xi^{h}=a \delta_{j}{ }^{h}+b J_{\jmath}{ }^{h}+\alpha_{j} \xi^{h}+\tilde{\alpha}_{j} \tilde{\xi}^{h},
$$




$$
\begin{array}{cc}
(5.2)^{\prime} & \nabla_{j} \tilde{\xi}^{h}=a J_{j}{ }^{h}-b \delta_{j}{ }^{h}+\alpha_{j} \tilde{\xi}^{h}-\tilde{\alpha}_{j} \xi^{h}, \\
(5.3) & \nabla_{k} \nabla_{j} \xi^{h}=a_{k} \delta_{\jmath}{ }^{h}+b_{k} J_{\jmath}{ }^{h}+\nabla_{k} \alpha_{j} \xi^{h}+\nabla_{k} \tilde{\alpha}_{\jmath} \tilde{\xi}^{h} \\
& +\alpha_{j}\left(a \delta_{k}{ }^{h}+b J_{k}{ }^{h}+\alpha_{k} \xi^{h}+\tilde{\alpha}_{k} \tilde{\xi}^{h}\right)+\tilde{\alpha}_{j}\left(a J_{k}{ }^{h}-b \delta_{k}{ }^{h}+\alpha_{k} \tilde{\xi}^{h}-\tilde{\alpha}_{k} \xi^{h}\right), \\
(5.4) & R_{k \jmath r}{ }^{h} \xi^{r}=X_{k} \delta_{j}{ }^{h}-X_{j} \delta_{k}{ }^{h}+Y_{k} J_{j}{ }^{h}-Y_{\jmath} J_{k}{ }^{h}+\alpha_{k j} \xi^{h}+\tilde{\alpha}_{k j} \tilde{\xi}^{h}, \\
(5.5) & X_{k}=a_{k}-a \alpha_{k}+b \tilde{\alpha}_{k}, \quad Y_{k}=b_{k}-b \alpha_{k}-a \tilde{\alpha}_{k},
\end{array}
$$

where we have put

$$
\alpha_{k \jmath}=\nabla_{k} \alpha_{\jmath}-\nabla_{\jmath} \alpha_{k}, \quad \tilde{\alpha}_{k \jmath}=\nabla_{k} \tilde{\alpha}_{j}-\nabla_{j} \tilde{\alpha}_{k} .
$$

Hence using (5.4) and the Bianchi identity $R_{k j i}{ }^{h}+R_{j \imath k}{ }^{h}+R_{\imath k j}{ }^{h}=0$, we have

$$
\begin{aligned}
& 2\left(Y_{k} J_{j h}+Y_{\jmath} J_{h k}+Y_{h} J_{k \jmath}\right) \\
& \quad+\alpha_{k \jmath} \xi_{h}+\alpha_{j h} \xi_{k}+\alpha_{h k} \xi_{\jmath}+\tilde{\alpha}_{k j} \tilde{\xi}_{h}+\tilde{\alpha}_{j h} \tilde{\xi}_{k}+\tilde{\alpha}_{h k} \tilde{\xi}_{\jmath}=0 .
\end{aligned}
$$

By the way, taking account of

$$
g_{r i} \underset{\xi}{L}\left\{\begin{array}{c}
r \\
\jmath
\end{array}\right\}=\nabla_{k} \nabla_{\jmath} \xi_{2}+R_{r k j i} \xi^{r},
$$

we have

$$
\begin{aligned}
& g_{r i} L\left\{\begin{array}{c}
r \\
j
\end{array}\right\}=a_{k} g_{j i}+a_{j} g_{k i}-X_{\imath} g_{j k}-Y_{\imath} J_{\jmath k}-b_{k} J_{\imath \jmath} \\
& +\left(b_{j}-2 b \alpha_{j}-2 a \tilde{\alpha}_{j}\right) J_{i k}+\alpha_{j i} \xi_{k}+\tilde{\alpha}_{j i} \tilde{\xi}_{k} \\
& +\left(\nabla_{k} \alpha_{\jmath}+\alpha_{j} \alpha_{k}-\tilde{\alpha}_{j} \tilde{\alpha}_{k}\right) \xi_{\imath}+\left(\nabla_{k} \tilde{\alpha}_{\jmath}+\alpha_{j} \tilde{\alpha}_{k}+\tilde{\alpha}_{\jmath} \alpha_{k}\right) \tilde{\xi}_{\imath},
\end{aligned}
$$

because of (5.3) and (5.4), where $L$ denotes the Lie derivation with respect to $\xi$. Since our manifold $M$ is Kaehlerian and $\xi$ is analytic, it is well known that

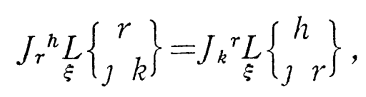

from which, using (5.9), we get

$$
\begin{aligned}
& Z_{k} g_{\jmath h}-Z_{h} g_{\jmath h}+\tilde{Z}_{h} J_{k j}-\tilde{Z}_{k} J_{h \jmath} \\
& \quad+J_{k}{ }^{r} u_{r j} \tilde{\xi}_{h}-J_{h}{ }^{r} u_{r j} \tilde{\xi}_{k}+u_{k j} \xi_{h}-u_{h j} \xi_{k}=0,
\end{aligned}
$$

where we have put

$$
Z_{k}=a_{k}+\tilde{b}_{k}, \quad u_{k \jmath}=\nabla_{k} \alpha_{\jmath}+J_{k}^{r} J_{\jmath}{ }^{s} \nabla_{r} \alpha_{s} .
$$

Again, changing $k, \jmath, \imath$ cyclically in (5.9) and adding those two obtained to (5.9), we get 


$$
\begin{aligned}
& 2\left(\tilde{Z}_{h} J_{k \jmath}+\tilde{Z}_{k} J_{j h}+\tilde{Z}_{\jmath} J_{h k}\right) \\
& +J_{k}^{r} u_{r j} \tilde{\xi}_{h}+J_{j}^{r} u_{r h} \tilde{\xi}_{k}+J_{h}{ }^{r} u_{r k} \tilde{\xi}_{j}-J_{h}^{r} u_{r j} \tilde{\xi}_{k}-J_{k}{ }^{r} u_{r h} \tilde{\xi}_{j}-J_{\jmath}{ }^{r} u_{r k} \tilde{\xi}_{h} \\
& +u_{k j} \xi_{h}+u_{j h} \xi_{k}+u_{h k} \xi_{j}-u_{h j} \xi_{k}-u_{k h} \xi_{j}-u_{j k} \xi_{h}=0 .
\end{aligned}
$$

In the next place, we assume that the associated form $\alpha$ is gradient, that is, it satisfies $\alpha_{i}=\nabla_{i} \alpha$. (This condition is established in any $K$-torse-forming vector field in a space of constant holomorphic sectional curvature. (See Proposition 2)). So the equations (5.7) and (5.11) can be rewritten as follows:

$$
\begin{gathered}
2\left(Y_{k} J_{j h}+Y_{\jmath} J_{h k}+Y_{h} J_{k j}\right) \\
+J_{k}{ }^{r} u_{r j} \tilde{\xi}_{h}+J_{\jmath}{ }^{r} u_{r h} \tilde{\xi}_{k}+J_{h}{ }^{r} u_{r k} \tilde{\xi}_{j}=0, \\
\tilde{Z}_{h} J_{k \jmath}+\tilde{Z}_{k} J_{j h}+\tilde{Z}_{\jmath} J_{h k}+J_{k}{ }^{r} u_{r j} \tilde{\xi}_{h}+J_{\jmath}{ }^{r} u_{r h} \tilde{\xi}_{k}+J_{h}{ }^{r} u_{r k} \tilde{\xi}_{j}=0,
\end{gathered}
$$

because of $J_{k}{ }^{r} u_{r j}+J_{\jmath}{ }^{r} u_{r k}=0$ and $u_{j k}=u_{k \jmath}$, and consequently

$$
\left(2 Y_{k}-\tilde{Z}_{k}\right) J_{j h}+\left(2 Y_{j}-\tilde{Z}_{j}\right) J_{h k}+\left(2 Y_{h}-\tilde{Z}_{h}\right) J_{k j}=0 .
$$

This together with (5.6) and (5.10) gives

$$
b_{k}+\tilde{a}_{k}=2\left(a \tilde{\alpha}_{k}+b \alpha_{k}\right) \text {, }
$$

or equivalently

$$
a_{k}-\tilde{b}_{k}=2\left(a \alpha_{k}-b \tilde{\alpha}_{k}\right)
$$

Thus we obtain

$$
X_{k}=-\tilde{Y}_{k} .
$$

Also, it follows from (5.14) and $(5.14)^{\prime}$ that

$$
a a_{k}+b b_{k}+b \tilde{a}_{k}-a \tilde{b}_{k}=2\left(a^{2}+b^{2}\right) \alpha_{k} .
$$

In the third place, suppose that $\xi$ is an analytic proper $K$-torse-forming vector field. Then (5.16) gives

$$
\nabla_{k}\left(\frac{1}{4} \log \left(a^{2}+b^{2}\right)-\alpha\right)=\frac{-b \tilde{a}_{k}+a \tilde{b}_{k}}{a^{2}+b^{2}},
$$

because of $\alpha_{i}=\nabla_{i} \alpha$. On the other hand, contracting (5.9) with $g^{h_{j}}$, $\xi^{h}$ and $\xi^{h} J^{k J}$ and taking account of $J_{k}{ }^{r} u_{r}+J_{\jmath}{ }^{r} u_{r k}=0$ and $u_{j k}=u_{k \jmath}$, we obtain respectively

$$
\begin{gathered}
(n-1)\left(a_{k}+\tilde{b}_{k}\right)+u_{k r} \xi^{r}-\nabla_{r} \alpha^{r} \xi_{k}=0, \\
|\xi|^{2} u_{k j}-u_{r j} \xi^{r} \xi_{k}-\tilde{\xi}^{r} u_{r j} \tilde{\xi}_{k}+\left(a_{k}+\tilde{b}_{k}\right) \xi_{j} \\
-\left(a_{r}+\tilde{b}_{r}\right) \xi^{r} g_{j k}+\left(b_{r}-\tilde{a}_{r}\right) \xi^{r} J_{k j}-\left(b_{k}-\tilde{a}_{k}\right) \tilde{\xi}_{j}=0,
\end{gathered}
$$




$$
\left(b_{r}-\tilde{a}_{r}\right) \xi^{r}=0 .
$$

Further, transvecting (5.8) with $\xi^{\prime}$ and using (5.19), we have

$$
|\xi|^{2} u_{k j} \xi^{\jmath}=\left[u_{r s} \xi^{r} \xi^{s}+\left(a_{r}+\tilde{b}_{r}\right) \xi^{r}\right] \xi_{k}-\left(a_{k}+\tilde{b}_{k}\right)|\xi|^{2},
$$

from which, comparing this with (5.17)

$$
a_{k}+\tilde{b}_{k}=2 \rho \xi_{k},
$$

or equivalently

$$
b_{k}-\tilde{a}_{k}=-2 \rho \tilde{\xi}_{k}
$$

for a certain function $\rho$. By virtue of $(5.16),(5.20)$ and $(5.20)^{\prime}$, it is clear that

$$
\frac{1}{2} \nabla_{k} \log \left(a^{2}+b^{2}\right)-\alpha_{k}=\frac{\rho}{a^{2}+b^{2}}\left(a \xi_{k}-b \tilde{\xi}_{k}\right) .
$$

Here we put

$$
f=\frac{1}{2} \log \left(a^{2}+b^{2}\right)-\alpha .
$$

Then, applying $\nabla$, to (5.22) and using (5.21), we find

$$
f_{j}=\frac{\rho}{a^{2}+b^{2}}\left(a \xi_{j}-b \tilde{\xi}_{j}\right),\left(f_{j}=\nabla_{\jmath} f\right),
$$

or equivalently

$$
\tilde{f}_{j}=\frac{\rho}{a^{2}+b^{2}}\left(a \tilde{\xi}_{j}+b \xi_{j}\right)
$$

So we have just shown that

$$
a f_{j}+b \tilde{f}_{j}=\rho \xi_{j}
$$

or equivalently

$$
a \tilde{f}_{j}-b f_{j}=\rho \tilde{\xi}_{j} .
$$

$\S 6$. $f$-hypersurfaces. Let $\xi$ be an analytic proper $K$-torse-forming vector field whose associated form $\alpha$ is locally gradient. A point $P$ of $M$ is called an ordinary point of $\xi$, if both of $\xi$ and $f_{h}$ given by (5.23) do not vanish at $P$. Let $M_{1}$ be the set of all ordinary points of $M$. Then $M_{1}$ is a non-empty open subset of $M$. We also see from (5.24) that $\rho$ has not zero points over $M_{1}$.

In the sequel we perform our discussions in $M_{1}$. Differentiating (5.23) covariantly and making use of (5.16), (5.21) $(5.24)$ and $(5.23)^{\prime} \sim(5.24)^{\prime}$, we find

$$
\nabla_{k} f_{j}=\rho g_{k}+\left(\nabla_{k} \log \rho-f_{k}\right) f_{\jmath}+\tilde{f}_{k} \tilde{f}_{\jmath},
$$

from which 


$$
\nabla_{k} \log \rho=\lambda f_{k}
$$

for a certain function $\lambda$, since $f$, is gradient. Thus (6.1) can be rewritten as follows :

$$
\nabla_{k} f_{\jmath}=\rho g_{k \jmath}+(\lambda-1) f_{k} f_{\jmath}+\tilde{f}_{k} \tilde{f}_{\jmath} .
$$

In a sufficiently small neighborhood of an ordinary point we consider the integral curve of the vector field $f^{h}$. By means of (6.3), we can easily find that such an integral curve is a geodesic arc.

Let $Q$ be an ordinary point in $M$ and $U$ a coordinate neighborhood of $Q$ which contain only ordinary points. So we can define in $U$ a family of hypersurfaces by the equations $f(x)=$ constant which will be called $f$-hypersurface. Given a point in $M_{1}$, there exists in the family one and only one $f$-hypersurface $V(P)$ passing $P$. It is clear that the $f$-curves form the normal congruence to the family of the $f$-hypersurfaces in $U$.

Put

$$
C^{h}=\frac{1}{\sigma} f^{h}, \quad \sigma=\sqrt{f_{r} f^{r}}
$$

in $M_{1}$, then $C^{h}$ is differentiable in $M_{1}$. As this equation and (6.3) yield that

$$
\nabla_{k} \sigma C_{\jmath}+\sigma \nabla_{k} C_{\jmath}=\rho g_{k \jmath}+(\lambda-1) \sigma^{2} C_{k} C_{\jmath}+\sigma^{2} \tilde{C}_{k} \tilde{C}_{\jmath},
$$

we get by transvection of this with $C^{\jmath}$

$$
\nabla_{k} \sigma=\left(\rho+(\lambda-1) \sigma^{2}\right) C_{k}
$$

which implies

$$
\nabla_{k} C_{\jmath}=\frac{\rho}{\sigma}\left(g_{k \jmath}-C_{k} C_{j}\right)+\sigma \tilde{C}_{k} \tilde{C}_{\jmath} .
$$

Let $P$ be a point in $U$ and $V(P)$ the $f$-hypersurfaces in $U$ passing through the point $P$. Then the vector field $C^{h}$ is the normal unit vector to $V(P)$ at any point of $V(P)$. We choose a system of local coordinates $\left\{u^{a}\right\}$ in $V(P)$ and suppose that $V(P)$ is expressed by parametric equations $x^{h}=x^{h}\left(u^{a}\right)$ in $U$. We notice that the second fundamental form $h$ of the $f$-hypersurface $V(P)$ is given by

$$
h_{a b}=B_{a}{ }^{\imath} B_{b}^{j} \nabla_{j} C_{\imath} .
$$

By virtue of (2.5) and (6.5), it is evident that

$$
h_{a b}=\frac{\rho}{\sigma} g^{\prime}{ }_{a b}+\sigma \eta_{a} \eta_{b} .
$$

So we can see that $V(P)$ is nothing but contact umbilic. By virtue of (6.2) and (6.4), we find respectively $\partial_{a} \rho=0$ and $\partial_{a} \sigma=0$ and consequently, we see that the functions $\rho$ and $\sigma$ are constant over $V(P)$. 
Now we can choose a system of coordinates $\left\{x^{h}\right\}$ in $U$ such that $f$-hypersurfaces defined by $x^{2 n}=$ constant are the $f$-hypersurfaces in $U$ and the curves defined by the equations $x^{a}=$ constant are the $f$-curves in $U$. Then it is easy to see that

$$
g_{a 2 n}=g_{2 n a}=0 .
$$

Since the $f$-curves are geodesics, we have

$$
\left\{\begin{array}{cc}
h \\
2 n & 2 n
\end{array}\right\}=\gamma \delta_{2 n}{ }^{h},
$$

where $\gamma$ is in $U$ a function depending only on $x^{2 n}$. Especially, if we put $h=a$, then it follows that

$$
\left\{\begin{array}{cc}
a \\
2 n & 2 n
\end{array}\right\}=0
$$

Recalling $g_{a 2 n}=0$ and $g^{a 2 n}=0$, we have

$$
\partial_{a} g_{2 n 2 n}=0 \text {, }
$$

which means that $g_{2 n}$ depends only on $x^{2 n}$. Hence, taking a suitable transformation of the $2 n$-th coordinate, we have $g_{2 n}=1$ in $U$. Then we find explicitly

$$
\left\{\begin{array}{cc}
h \\
2 n & 2 n
\end{array}\right\}=0 \text {. }
$$

And the variable $x^{2 n}$ is the arc-length of $f$-curves in $U$. So the line element of the Kaehlerian manifold $M$ is written in the form

$$
d s^{2}=g_{a b}\left(x^{h}\right) d x^{a} d x^{b}+\left(d x^{2 n}\right)^{2} .
$$

Thus we get

THEOREM 4. If a Kaehlerian manifold $M$ admits an analytic proper K-torseforming vector field $\xi$ such that the associated form is locally gradient, then for any ordinary point $P$ of the vector field $\xi$, there exists a coordinate neighborhood $U$ of the point $P$ in such a way that there is in $U$ a system of coordinates $\left\{x^{h}\right\}$ having the following properties The function $f$ depends only on the $2 n$-th variable $x^{2 n}$ in $U$. The line element of $M$ is given by (6.7) in $U$. The hypersurfaces defined by the equation $x^{2 n}=$ constant are the f-hypersurfaces and the curves defined by the equation $x^{a}=$ constant are the $f$-curves and $x^{2 n}$ indicates the arc length along the f-curves. Moreover, f-hypersurfaces are contact umbilic.

Conversely, we assume that in a Kaehlerian manifold $M$ there exists a coordinate neighborhood $U$ in $M$ such that there exists family of contact umbilical hypersurfaces

$$
f\left(x^{h}\right)=\text { constant }
$$


whose orthogonal trajectories are geodesics. Then operating $\nabla^{\prime}{ }_{a}$ to (6.8), we can easily find that

$$
f_{h} B_{a}{ }^{h}=0 .
$$

Furthermore, differentiation of the above equation gives

which means that

$$
\nabla_{k} f_{\jmath} B_{a}{ }^{k} B_{b}{ }^{j}+f_{k} C^{k} h_{a b}=0,
$$

$$
\left[\nabla_{k} f_{\jmath}+f_{r} C^{r}\left(\alpha g_{k}+\beta J_{k h} C^{h} J_{\jmath m} C^{m}\right)\right] B_{b}{ }^{k} B_{a}{ }^{j}=0,
$$

because $h_{a b}=\alpha g^{\prime}{ }_{a b}+\beta \eta_{a} \eta_{b}$, where $C^{h}$ denotes the unit normal vector of the hypersurface. Consequently we see that $\nabla_{k} f$, must take the form

$$
\nabla_{k} f_{j}=\rho g_{k \jmath}+a f_{k} f_{\jmath}+b \tilde{f}_{k} \tilde{f}_{\jmath},
$$

or equivalently

$$
\nabla_{k} \tilde{f}_{\jmath}=\rho J_{k \jmath}+a f_{k} \tilde{f}_{\jmath}-b \tilde{f}_{k} f_{\jmath}
$$

for certain functions $\rho, a$ and $b$. If we put

$$
\xi^{h}=c f^{h}+e f^{h}
$$

for any functions $c$ and $e$ such that $c^{2}+e^{2} \neq 0$, then we have

$$
\nabla_{j} \xi^{h}=c \rho \delta_{j}{ }^{h}+e \rho J_{j}{ }^{h}+\alpha_{j} \xi^{h}+\beta_{j} \tilde{\xi}^{h},
$$

$\alpha$, and $\beta$, being certain 1 -forms. The above equation means that $\xi$ is a $K$-torseforming vector field. Therefore we have

THEOREM 5. If there exists a coordinate neighborhood $U$ in a Kaehlerian manifold $M$ such that there exists a family of contact umbilical hypersurfaces whose orthogonal trajectories are geodesics, then there exists a K-torese-forming vector field in $U$.

§7. Examples. In [5] we have proved that in order that a Kaehlerian manifold $M$ is holomorphically subprojective, it is necessary and sufficient that there exists a local coordinate system $\left\{x^{h}\right\}$ such that the Christoffel symbols $\left\{\begin{array}{c}h \\ j\end{array}\right\}$ of $M$ take the form

$$
\begin{gathered}
\left\{\begin{array}{c}
h \\
j \quad i
\end{array}\right\}=\rho_{j} \delta_{i}{ }^{h}+\rho_{i} \delta_{\jmath}{ }^{h}+\tilde{\rho}_{\jmath} J_{i}{ }^{h}+\tilde{\rho}_{\imath} J_{\jmath}{ }^{h}+f_{j i} x^{h}-f_{\jmath r} J_{i}{ }^{r} \tilde{x}^{h} \\
f_{[j k]}=0, \quad f_{r[\jmath} J_{i]}{ }^{h}=0,
\end{gathered}
$$

where $\rho_{\imath}$ and $f_{j k}$ are 1 -form and a covariant tensor field respectively. Now, consider a vector field $V$ such that $V$ are given by $V^{h}=x^{h}$ with respect to a 
sysyem of coordinate $\left\{x^{h}\right\}$ having the properties above mentioned. Differentiate it covariantly with respect to the connection (7.1), we have by virtue of (7.2)

$$
\nabla, V^{h}=\left(1+\rho_{r} V^{r}\right) \delta_{\jmath}{ }^{h}+\tilde{\rho}_{r} V^{r} J_{\jmath}{ }^{h}+\alpha_{\jmath} V^{h}+\tilde{\alpha}_{\jmath} \tilde{V}^{h},
$$

where we have put $\alpha_{\jmath}=\rho_{\jmath}+f_{\jmath r} V^{r}$. Moreover we have proved in [5] that the associated form is gradient. These facts tell us that the vector field $V$ is nothing but an analytic $K$-torse-forming vector field whose associated form is gradient.

In [5, III] we have also shown that the Christoffel symbols of the holomorphically subprojective Kaehlerian manifold of the first kind take the form

$$
\left\{\begin{array}{c}
h \\
j \quad i
\end{array}\right\}=\rho_{j} \delta_{i}{ }^{h}+\rho_{i} \delta_{J}{ }^{h}+\tilde{\rho}_{\imath} J_{J}{ }^{h}+\tilde{\rho}_{J} J_{i}{ }^{h}+f_{J i} \xi^{h}-f_{\jmath r} J_{i}{ }^{r} \tilde{\xi}^{h},
$$

for suitable coordinate system $\left\{x^{h}\right\}$, where $f_{[j k]}=0, f_{r\lfloor\jmath} J_{i]}{ }^{h}=0$ and $\xi^{h}$ is an analytic $K$-torse-forming vector field whose associated form is gradient.

The author wishes to express his sincere thanks to Professor K. Yano who gave personal discussions, and also to Professors S. Tachibana and S. Ishihara who gave criticisms and kind advices.

\section{BIBLIOGRAPHY}

[1] Ishinara, S., On infinitesimal concircular transformations, Kōdar Math. Sem. Rep., 12 (1960), 45-56.

[2] ISHIHARA S. AND Y. TASHIRO, On Riemannian manifolds admitting a concircular transformations, Math. J. Okayama Univ., 9 (1959), 19-48.

[3] TASHIRo, Y., On conformal and projective transformations in Kählerian manifolds, Tôhoku Math. J., 14 (1962), 317-320.

[4] Tashiro Y. And S. Tachibana, On Fubınian and C-Fubınıan manifolds, Kōdai Math. Sem. Rep., 15 (1963), 176-183.

[5] Yamaguchi S. AND T, Adati, On holomorphically subprojective Kählerian manifolds, I, II, III, Ann di Mate. pura ed appli., 112 (1777), 217-229, Accad. Naz. dei Lincei, 60 (1976), 405-413, Ann di Mate. pura ed appli., 113 (1977), 111-125.

[6] Yano, K., Concircular geometry, I, II, III, IV, V, Proc. Imp. Acad. Tokyo, 16 (1940), 195-200, 354-360, 442-448, 505-511, 18 (1942), 446-451.

[7] Yano, K., Sur le parallelism et le concurrence dans l'espace, Proc. Imp. Acad. Tokyo, 20 (1943), 189-197.

[8.] Yano, K., On the torse-forming directions in Riemannian spaces, Proc. Imp. Acad. Tokyo, 20 (1944), 340-345.

[9] Yano, K., Differential geometry on complex and almost complex spaces, Pergamon Press, Oxford, 1965.

[10] YANO K. AND S. IshinARA, Almost contact structure induced on hypersurfaces in complex and almost complex spaces, Kōdai Math. Sem. Rep., 17 (1965), $222-249$.

Department of Mathematics

Science University of Tokyo

TOKYO, JAPAN 